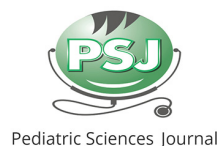

Published by

Pediatrics Sciences Journal

\section{Sphingomonas paucimobilis an unusual cause of subdural empyema in pediatric: a case report}

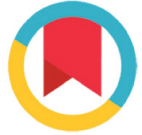

CrossMark

\author{
Annisa Muhyi ${ }^{1}$, Amalia Aswin ${ }^{2 *}$
}

ABSTRACT

'Department of Child Health, Mulawarman University, Abdul Wahab Sjahranie Hospital, Samarinda, Indonesia ${ }^{2}$ Faculty of Medicine, Mulawarman University, Samarinda, Indonesia

\section{*Corresponding to:}

Amalia Aswin; Faculty of Medicine,

Mulawarman University;

x11amaliaaswin@gmail.com

\section{Received: 2021-03-16}

Accepted: 2021-05-20

Published: 2021-06-01
Introduction: Acute bacterial meningitis continues to be a neurological emergency with high mortality and morbidity Case: We report the case of a pediatric patient with subdural empyema due to Sphingomonas paucimobilis. A 2-month-old infant developed fever, generalized seizures, and worsening general status. He had a cough for two weeks, and a chest X-ray showed pneumonia. Laboratory tests reveal marked leukocytosis, a white blood cell count of $28.500 / \mathrm{mL}$, and an elevated platelet level of $654.000 \mathrm{mg} / \mathrm{dL}$. A computerized brain tomography revealed an empyema subdural. A cerebrospinal fluid analysis showed unclear fluid, revealed cell count 3560 cells, $90 \%$ mononuclear and 10\% polymorphonuclear, red blood cell count of $0 / \mathrm{mm} 3$, protein of $370 \mathrm{mg} / \mathrm{dL}$, and glucose of $35 \mathrm{mg} / \mathrm{dL}$. His cerebrospinal fluid culture showed Sphingomonas paucimobilis sensitive to cephalosporin, levofloxacin, meropenem, imipenem, gentamycin, tobramycin and amikacin. He was treated empirically with meropenem. The patient had a rapid recovery from the seventh day of treatment and was discharged on hospital day 14.

Conclusion: We highly recommend raising awareness about subdural empyema as a complication for acute bacterial meningitis caused by $S$. paucimobilis in community-acquired infection.

Keywords: Sphingomonas paucimobilis; subdural empyema; meningitis; case report.

Cite This Article: Muhyi, A., Aswin, A. 2021. Sphingomonas paucimobilis an unusual cause of subdural empyema in pediatric: a case report. Pediatrics Sciences Journal 2(1): 1-3.

\section{INTRODUCTION}

Sphingomonas paucimobilis is an aerobic, yellow-pigmented, glucosenonfermenting bacterium, gram-negative bacillus, characterized by catalase and oxidative activities. ${ }^{1,2}$ S. paucimobilis can be found in seawater, sea ice, river water, wastewater, mineral water, soil, medical equipment, and tools at hospitals, including hospital water system, respiratory assist devices, and laboratory instrument. ${ }^{2,3}$ This organism has been identified in multiple organs such as septic arthritis, osteomyelitis, urinary tract infection, diarrheal disease, bacteremia, cutaneous infections, biliary tract infections, ventilator-associated pneumonia, peritonitis, and few patients with brain structure involvement. ${ }^{4}$ Only 5 cases of meningitis caused by this organism have been reported so far, and most of them are adults.

Acute bacterial meningitis continues to be a neurological emergency with high mortality and morbidity. ${ }^{5}$ The most common neurologic complication found in a patient with meningitis is cerebral edema and increase intracranial pressure, along with ventriculitis, seizures, cerebral infarction, subdural empyema or effusion, hydrocephalus, hearing loss, intellectual disability, and developmental delay. ${ }^{6}$ Subdural empyema is a less common lifethreatening complication of meningitis and is only found in $2.7 \%$ of communityacquired bacterial meningitis cases. ${ }^{7}$ We at this moment report on an unusual case of subdural empyema as a complication of acute bacterial meningitis caused by Sphigomonas paucimobilis.

\section{CASE REPORT}

A 2-month-old infant was admitted to the emergency department with convulsive status epilepticus with fever. He got generalized seizures and a worsening general status. No history of seizures without fever. On neurological examination, the patient was irritable, pupils were normal, light reflex $+/+$, and showed upper motor neuron lesion. The Glasgow Coma Scale (GCS) was $13 / 15$. There were no signs of meningeal irritation or stiff neck. Laboratory tests reveal marked leukocytosis, a white blood cell count of $28,500 / \mathrm{mL}$, and an elevated platelet level of $654.000 \mathrm{mg} / \mathrm{dL}$. $\mathrm{He}$ otherwise is found to have normal hemoglobin, blood glucose, electrolyte levels, and regular hepatic and renal studies. The thorax x-ray was found without any abnormality (Figure 1). A computerized brain tomography revealed an empyema

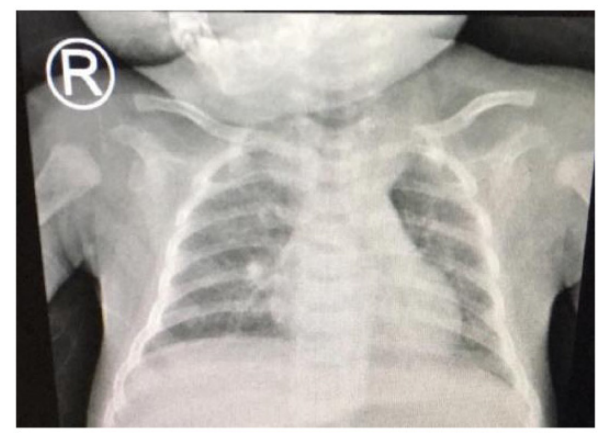

Figure 1. Thorax x-ray 


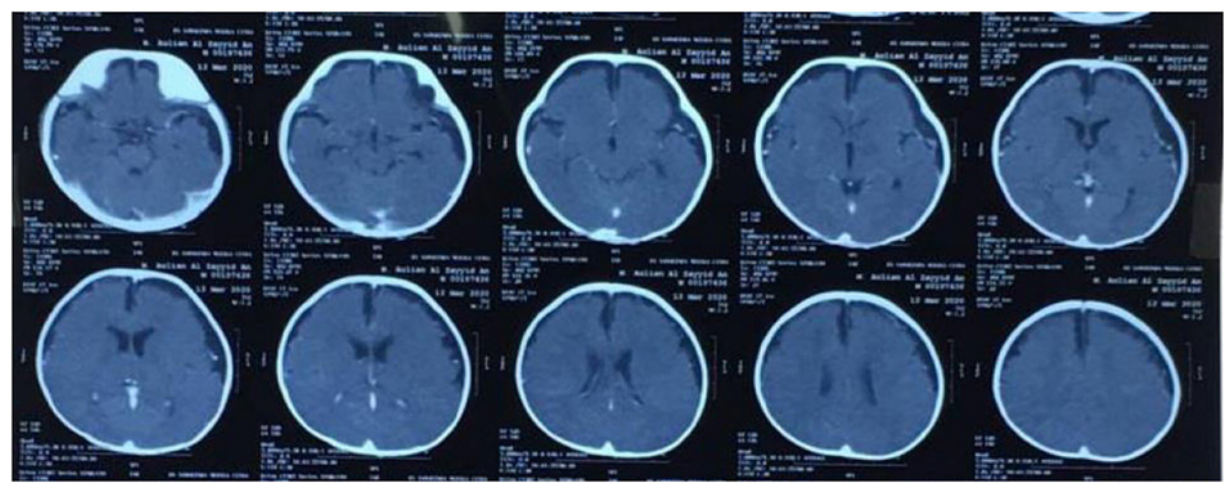

Figure 2. Head computerized tomography scan showed empyema subdural

subdural (Figure 2). A cerebrospinal fluid analysis showed unclear fluid, revealed cell count 3560 cells, mononuclear $90 \%$ and polymorphonuclear $10 \%$, red blood cell count of $0 / \mathrm{mm} 3$, protein of $370 \mathrm{mg} / \mathrm{dL}$, and glucose of $35 \mathrm{mg} / \mathrm{dL}$. His cerebrospinal fluid culture showed Sphingomonas paucimobilis sensitive to cephalosporin, levofloxacin, meropenem, imipenem, gentamycin, tobramycin, and amikacin. He was treated with meropenem for a total of 14 days. He underwent burr-hole evacuation surgery by the neurosurgeon. $\mathrm{He}$ showed significant improvement after seven days and was subsequently discharged home after complete 14 days of intravenous meropenem. Total blood count returned to typical values the next day. He was discharged from the hospital on day 14.

We evaluated growth and development every month after discharged from the hospital. During outpatient care, there is no complaint of seizure, fever, and neurological disorders.

\section{DISCUSSION}

Sphingomonas paucimobilis is an opportunistic pathogen with sphingoglycolipid in the cell wall and lacks the lipopolysaccharide component and its endotoxin activity. ${ }^{2}$ It rarely causes infection because of its relatively low virulence. ${ }^{8}$ S. paucimobilis has a wide range of biodegradative abilities and is considered an oligotrophic bacterium, meaning it can live in an environment with low nutrient content. ' Infection of S. paucimobilis could be community and hospital acquired. The sterile water supply system and hospital, the distilled water, hemodialysis fluids, sterile medicine preparation solution can easily be contaminated with S. paucimobilis. ${ }^{3} \mathrm{~S}$. paucimobilis has been isolated from a wide variety of clinical specimens, including blood, urine, sputum, cerebrospinal fluid. ${ }^{9}$ It has been reported in a type of infection from primary bacteremia to lifethreatening meningitis, peritonitis, and septic shock. ${ }^{1}$

Subdural empyema is a neurosurgical emergency that requires prompt recognition and treatment. ${ }^{10}$ The most common cause of subdural empyema in infants is meningitis. ${ }^{10}$ The source of infection for subdural empyema varies according to patient age. In the infant, the common source was secondary infection from bacterial meningitis, while sinusitis and otitis media are the primary sources of infection in older children. ${ }^{10,11}$ The male predominance in the present series is in concordance with that in the previous pediatric series. ${ }^{12}$ Ryan and Adley (2010) concluded that empyema comprised $2 \%$ of S. paucimobilis infections. The majority of patients present with two out four following symptoms: headache, fever, neck stiffness, and altered mental status (Glasgow Coma Scale $<14)^{7}$ Hendaus (2013) stated $40 \%$ of patients with subdural empyema present with seizures. ${ }^{13}$ Older children can present with symptoms of primary pathology such as sinusitis or otitis, fever, headache, photophobia, purulent rhinorrhea, and painful paraesthesiae over the face. ${ }^{10}$ In our case, the patient is an infant with fever, altered mental status (GCS 13), and seizures.

Essential clues for the diagnosis of empyema were otitis or sinusitis, focal neurologic deficits, or epileptic seizures. ${ }^{14}$ Laboratory tests will usually demonstrate leukocytosis with shift to the left, elevated C-reactive protein, and erythrocyte sedimentation rate. ${ }^{10}$ Cranial sonography to differentiate subdural empyema from subdural effusion. Plain skull and sinus radiographs can reveal a skull fracture, opacification of sinuses, osteomyelitis, or a lodged foreign body or diastasis of the sutures in an infant. ${ }^{10}$ Computed Tomography with high-resolution contrast may be expected in up to $50 \%$ of patients or only show non-specific hemisphere swelling and minimal midline shift. It will show crescentic or lentiform extra-axial hypodense collections with prominent, sharp medial rim enhancement. ${ }^{10}$

Bacterial meningitis is considered a significant source of subdural empyema in infants, and cerebrospinal fluid is the gold standard for diagnosis. ${ }^{13}$ Infants with bacterial meningitis have an accumulation of extra-axial fluid or subdural collection of pus that may turn to an empyema. If it is not treated early and properly, this condition may require surgical drainage. ${ }^{6}$

In our case, Sphingomonas paucimobilis was isolated from CSF culture. In the presence of an underlying disease and no risk factors, the most likely source of infection could be pneumonia. Previously it was known to produce nosocomial conditions, especially in the immunocompromised individual. Still, now an increasing frequency has been reported among healthy people of all age groups as community-acquired infection. ${ }^{3}$ To our knowledge, this is the first case report in the literature of subdural empyema as a complication of acute bacterial meningitis caused by S. paucimobilis infection in a pediatric department.

S. paucimobilis isolates are generally resistant to penicillin and the first generation of cephalosporins. ${ }^{3}$ The carbapenems were the most effective antibiotic therapy along with aminoglycosides, trimethoprimsulfamethoxazole, and piperacillin or tazobactam. ${ }^{8}$ The strain of S. paucimobilis isolated from our patient was sensitive to cephalosporin, levofloxacin, meropenem, imipenem, gentamycin, tobramycin, and amikacin. 
Although the antibiotic susceptibility for Sphingomonas paucimobilis is highly variable because $S$. paucimobilis can produce beta-lactamase, the majority of the studies showed sensitivity towards fluoroquinolones, beta-lactam combinations, and carbapenems. ${ }^{15} \mathrm{~A}$ study showed the most resistant pattern identified in children was against thirdgeneration cephalosporin (20. 9\%), ampicillin (12.5\%), and amikacin (8.2\%), while carbapenems were the most effective antibiotic therapy. As shown in this case, our patient was treated with meropenem. The patient was evaluated and managed on time, and a significant outcome was observed with meropenem for 14 days.

We, therefore, suggest that the most appropriate management for children with convulsive status epilepticus with fever is to start parenteral antibiotics early, perform a lumbar puncture when there are no contraindications, and start therapy based on cerebrospinal fluid findings. ${ }^{16}$ Early treatment of acute bacterial meningitis may decrease the complication, including subdural empyema. ${ }^{16}$

Since the pus in the subdural cavity can trigger epilepsy, anti-seizure medication is needed in the acute phase; the duration of anti-seizure is variable based on the positive history of seizure. ${ }^{10}$

The first modality of surgical therapy in children is twist drill hole aspiration, followed by craniotomy, burr hole evacuation, and endoscopic evaluation. ${ }^{10}$ Burr hole evacuations are usually indicated when the patient is in septic shock, emergency decompression, or in situations where the patient is considered very frail. ${ }^{10}$ Konar et al. (2019) reported $73.5 \%$ of cases of subdural empyema underwent craniotomy evacuation of pus and excision of the outer membrane, $26.5 \%$ cases underwent burr-hole evacuation of the pus collection. ${ }^{11}$

Neurological deficit and cerebral herniation are indicators of poor prognosis. The prognosis is good if the patient is treated early, with a survival rate of more than $90 \%$. The prognosis is terrible if the patient is younger than ten years, comatose on presentation, delayed in antibiotic therapy, and diffusely spread..$^{10}$

\section{CONCLUSION}

In conclusion, we present a case of subdural empyema in an infant with meningitis caused by an unusual organism. We highly recommend raising awareness about subdural empyema as a complication for acute bacterial meningitis caused by S. paucimobilis in community-acquired infection. Early diagnosis and treatment are essential for a gratifying outcome.

\section{ETHICS CONSIDERATION}

This case report has followed the ethics publication guideline by COPE and ICMJE as well as informed consent from the patient prior to the study being conducted.

\section{FUNDING}

None

\section{AUTHOR CONTRIBUTIONS}

All authors equally contribute to this case study from the data acquisition, data analysis, until reporting the results of study through publication.

\section{CONFLICT OF INTEREST}

The authors report no declarations of interest.

\section{REFERENCES}

1. Saboor F, Amin F, Nadeem S. Community acquired Sphingomonas paucimobilis in a child - A rare case. J Pak Med Assoc. 2018; 68(11): 1714-15.

2. Osuji A, Imaji J, Olateju E, Ekele B, Baamlong $\mathrm{N}$, Tahiru Y. Hospital-acquired Sphingomonas paucimobilis Infection in a Neonate: A Case Report. Journal of Advances in Medicine and Medical Research. 2020; 32(7): 42-46.
3. Deveci N, Gurkan N, Belet N, Baysal S. Sphingomonas paucimobilis: An Uncommon Cause of Meningitis. J Pediatr Inf. 2017; 11(3): 124-128.

4. Hernandez J, Vasquez A, Restrepo A. Sphingomonas paucimobilis Meningitis in a Child: First Case Report. EC Neurology. 2019; 11(2): 127-129.

5. Dakkak M, Cllinane W, Ramoutar V. Subdural Empyema Complicating Bacterial Meningitis: A Challenging Diagnosis in a Patient with Polysubstance Abuse. Case Report in Medicine. 2015;1-3.

6. Kather F, Set K. An Infant with Status Epilepticus and Stroke. Pediatrics in Review. 2019; 40(8): 431-434.

7. Alamarat Z, Hasbun R. Management of Acute Bacterial Meningitis in Children. Infection and Drug Resistance. 2020; 13: 4077-89.

8. Mehmood H, Khan N, Ullah S, Ullah A, Marwat A. A Rare Case of Sphingomonas paucimobilis Meningitis in the Absence of Cerebrospinal Fluid Pleocytosis. Journal of Investigative Medicine High Impact Case Reports. 2018; 6: 1-3.

9. Ryan MP, Adley CC. Sphingomonas paucimobilis: a persistent Gram-negative nosocomial infectious organism. Journal of Hospital Infection. 2010; 75: 153-157.

10. Muzumdar D, Biyani N, Deopujari C. Subdural empyema in children. Child's Nervous System. 2018

11. Jim K, Brouwer M, Ende Avd, Beek Dvd. Subdural empyema in bacterial meningitis. Neurology. 2012; 79: 2133-39.

12. Hendaus M. Subdural Empyema in Children. Global Journal of Health Science. 2013; 5(6): 54-57.

13. Konar S, Gohil D, Shukla D, Sadashiva N, Uppar A, Bhat D, et al. Predictors of outcome of subdural empyema in children. Journal of Neurosurgery. 2019; 47(2): 1-6.

14. Liu Z, Chen N, Tu P, Lee S, Wu C. The treatment and outcome of postmeningitic subdural empyema in infants. J Neurosurg Pediatrics. 2010; 6: 38-42.

15. Yuan J, Treadwell T. Sphingomonas paucimobilis empyema caused by remote foreign body aspiration. BMJ Case Reports. 2018; 1-3.

16. Chin RF, Neville BG, Scott RC. Meningitis is a common cause of convulsive status epilepticus with fever. Arch Dis Child. 2004; 90: 66-69.

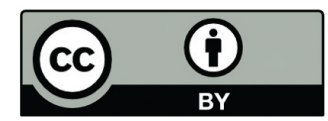

This work is licensed under a Creative Commons Attribution 